\title{
Forensic Malware Analysis: The Value of Fuzzy Hashing Algorithms in Identifying Similarities
}

\author{
Nikolaos Sarantinos*, Chafika Benzaïd ${ }^{\dagger}$, Omar Arabiat ${ }^{\ddagger}$ and Ameer Al-Nemrat* \\ * School of Architecture, Computing \& Engineering, UEL, London, UK \\ ${ }^{\dagger}$ Division Sécurité Informatique, CERIST, Alger, Algérie \\ ${ }^{\ddagger}$ AlBalqa Applied University
}

\begin{abstract}
This research aims to examine the effectiveness and efficiency of fuzzing hashing algorithm in the identification of similarities in Malware Analysis. More precisely, it will present the benefit of using fuzzy hashing algorithms, such as ssdeep, sdhash, mvHash and mrsh v2, in identifying similarities in Malware domain. The obtained results will be compared with the traditional and most common Cryptographic Hashes, such as the MD5, SHA-1 and SHA-256. Furthermore, it will highlight the pros and cons of fuzzy and cryptographic hashing, as well as their adoption in real world applications.
\end{abstract}

\section{INTRODUCTION}

Anti-virus vendors, researchers and analysts are struggling to keep up and to identify all the new malware types that are created every day. Malware Authors have managed to increase their malware's sophistication to avoid detection against anti-malware technics by "implementing new features and specific modifications, such as encryption, polymorphism and metamorphism to maximize their resilience." [1]. However, anti-virus vendors and analysts managed to adapt their identification techniques by relying "on automated analysis methods, and tools in order to distinguish malicious from benign code" [2], such as the traditional static analysis using Cryptographic hashes with the MD5 and SHA256 being "the most commonly used in Malware Research in 2013." [3]. Before pursuing further the presentation of the relevant literature review, with regards to static analysis and the hashing types, it is worth defining the different malware types and presenting their key characteristics and defense mechanisms used to avoid detection.

\section{HASH VALUES}

The hash values, which are also called digest or checksum, have been a standard procedure and the main tool used in the traditional static malware analysis, and generally in any digital forensic investigation. They have been used primarily for the purpose of "identification, verification and authentication of file data" [4]. They are widely spread because of their "two basic properties: the compression and ease of computation, where compression means that independent of the input length the output (hash value) is of fixed size." [5]. The output hash value is "the unique identifier for the acquired data, and is just as a DNA sequence or human fingerprint that identifies an individual" [4], governed investigation process by its three fundamental forensic hashing rules [6]:

1) You can't predict the hash value of a file or device;

2) No two-hash values can be the same; (Note: Collisions have occurred in research using supercomputers.)

3) If anything changes in the file or device, the hash value must change.

In order for malware analysts and forensics investigators to be able to detect the known malware and the "bad files" they make use of white and black list filters. The Whitelist is mainly a hash database, and it is used to reduce the amount of data that requires further analysis. More precisely, it means that, "if the file is 'known-to-be good', the analyst or investigator can fade out the file from further investigation." [7]. The Blacklist is the opposite of the Whitelist, which means that "if the file is 'known-to-be-bad', the analysts look at the file by hand and check, if it actually is illicit." [7]. However, the downside of using this approach is that any change in a single bit of a file or data means that their hash values will also change.

Finally, before discussing fuzzy hashing and its concept, we must understand the functions and the differences between the categories of hash algorithms, which are the Cryptographic, the Rolling, and Piecewise Hashing.

\section{CRYPTOGRAPHiC HASHES}

Cryptographic hashes have been the traditional tool, for both malware analysis and forensic investigations. According to Dunham [3] the most used Cryptographic Hashes for malware analysis to detect identical known malware signatures are the MD5, SHA-1, and SHA-256. The main difference between these Cryptographic Hashes is their hash value length, despite they share the same concept "to perform known file filtering" [8] by identifying identical matches.

As mentioned earlier, changing a single bit in a file/document means that its hash value will also change, which makes it impossible- arguably- to find any associations or similarities between two files or to check if they are virtually similar.

\section{A. Rolling Hashes}

The Rolling Hashes are generating 'pieces' of the traditional hash strings by "producing a pseudo random value based only 
on the context of the input," [9]. They are based on the Rabin-Karp algorithm, which is defined as: "Given a string $\mathrm{P}$ of length $\mathrm{N}$ and a string $\mathrm{S}$ of length $\mathrm{M}$ to find out all the occurrences of P within S." [10]. They are popular because they are easy and fast to compute. They are "used to identify similar strings in blocks of data" [11].

\section{B. Piecewise Hashing}

The Piecewise Hashing generates a final checksum for the whole document like traditional hashes. They overcome the limitations and the drawbacks of the latter as piecewise hashes separates the whole file into fixed segments/pieces, then generates a hash values for each of these segments. The generated segments values are, at the end, forming the final hash sequence. Furthermore, they were initially created to reduce the potential errors during the forensic imaging, so that the integrity of the data will be absolute, because only one hash segment would be void.

\section{Similarity - FUZZY HASHING}

Fuzzy hashing $(\mathrm{FH})$, which is also called Context Triggered Piecewise Hashing (CTPH), is a combination of Cryptographic Hashes $(\mathrm{CH})$, Rolling Hashes $(\mathrm{RH})$ and Piecewise Hashes (PH), and according to [7] it can be perceived as: $\mathrm{FH}=\mathrm{CTPH}$ $=\mathrm{PH}+\mathrm{RH}$. Unlike the traditional hashes, which their hashes (checksum) can be seen more as right or wrong, and as black or white, CTPH is more like the "grey hash type, as it can identify two files that may be near copies of one another that normally may not be located using traditional hashing methods." [3]. The identification between the two or more files is presented by their syntax similarity using a "score percentage between 0 and 100, where 0 is low similarity/probability and 100 is high similarity/probability [12].

The first version of CTPH was not initially meant for the use of malware detection. In 1999 Andrew Tridgell created the algorithm as a more effective and efficient way to find updates of files, which was later modified to identify/filter spam mails based on similarities and was called spamsum algorithm. Kornblum [9] in 2006 adjusted the Tridgell's spamsum "to cope with files and released ssdeep. His main idea was to compute cryptographic hashes not over the entire file, but over parts of it, which are called segments or chunks" [7]. According to Roussev [8], fuzzy hashing was developed due to some limitations of the Cryptographic Hashes and with the purpose of addressing the following boundaries:

\section{Identification of embedded/trace evidence}

Given a piece of data, such as a JPEG, an investigator needs to be able to search for (traces of) its existence inside another document, archive, disk image, or network trace.

\section{Identification of code versions}

Modern software is dynamically patched and upgraded on a daily basis; it is an infeasible to maintain crypto-hash inventory of all the files for every single version.

\section{Identification of related documents}

Many documents undergo changes/transformations as they are updated. It is often necessary to be able to identify and trace the versions across multiple evidence sources.

\section{Correlation of memory and disk sources}

An investigator needs to be able to correlate memory captures and disk images. The run-time layout and content of an executable/document are different from the on-disk representation so conventional hashes fail; however, identifiable commonality is clearly present.

\section{Correlation of network and disk sources}

Transmitted files are fragmented and interleaved. Currently, correlation requires time-consuming packet flow reconstruction and protocol parsing to extract transmitted files before any hash filtering can be applied.

However, the efficiency of Fuzzy Hashing to identify similarities between files depends only on one factor; it requires a 'sample' that can be "compared to something, and therefore having some sort of malware library greatly increases the effectiveness of using fuzzy hashes to determine if a suspect file is malicious or not" [13]. More precisely, this means that fuzzy hashing is only suitable for static and offline analysis, and the overall process can turned out to be time consuming. However, according to Breitinger [5], fuzzy hashing still remains a good and promising technique against metamorphic malware. The main four and common similarity hashing algorithms will be presented in the following subsections.

\section{A. ssdeep}

Kornblum's ssdeep (similarity digest) fuzzing tool was the first fuzzing algorithm created that resolved some of the margins of the block-bashed hashes. The algorithm follows three steps:

(i) It uses Rolling Hashing to split the document "into a 6 bit value segments" [12] (blocks of variable length, which are depended of the Rolling Hash Algorithm);

(ii) It uses another hash function, such as MD5 or SHA-1, to produce a digest for each segment;

(iii) It links together all hash segments to form the hash signature.

Although ssdeep is quite effective in finding similarities between text files, because it was initially created for spam detection, its detection rate for images and videos is low as an active adversary can exploit it.

\section{B. sdhash}

Roussev developed in 2010 the sdhash (Similarity Digest hash), which uses Bloom Filters to encode the output hash features identified with low empirical probability. Its results are based on a "similarity score by calculating a normalized entropy measure between the digests ... which ranges from 0 to 100 , where 0 is a mismatch and 100 is a perfect match or a near match" [12]. According to Roussev [8] (2011) and Breitinger and Baier [5], the advantage of sdhash is that the entropy calculation is performed for every 64-byte sequence (from 0 to 63 , then from 1 to 64 , etc.), and the identified features are hashed with SHA-1 and inserted into a Bloom Filter. This 
means that the sdhash can identify similarities between files on condition that common features are identified.

\section{C. mvHash}

The mvHash (majority vote hash) algorithm is a Similarity Preserving Digest (SPD), which has the "fastest computation time than any other SPH algorithm, and is almost as fast as SHA-I" [14]. The mvHash uses also the Bloom Filters, such as sdhash, and the "inputs are similar if they have similar underlying byte sequences" [14]. The algorithm has three phases: first the majority vote is made on a bit level so that any sequence will be transformed into 0 s and $1 \mathrm{~s}$. The second phase is the RLE (Run Length Encoding) and "is applied to represent these sequence of $0 s$ and 1 s by its length (in bytes)". The last phase is the creation of the similarity digest.

\section{D. $m r s h$ v2}

Multi-resolution similarity hashing (mrsh) is powerful variation of the ssdeep. The main difference between these two SPHs is that ssdeep is using the rolling hash, and mrsh is using a polynomial hash - djb2. Also, the mrsh uses the MD5 to compute the hashes, and "generates a variable sized similarity digest and adapts a technique from md5bloom that uses Bloom filters to represent the MD5 chunk hashes".

\section{Malware Detection with Hashing}

According to Sikorski and Honig [15], the first steps for identifying any malware are to use Anti-virus Scanning software and then calculate their hash values (i.e., Fingerprint the Malware). Although, Cryptographic Hashes can only be used to identify known malware, which have been already identified, based on their checksum, they are also used "for documentation and future integrity verification" [16]. Furthermore, that way we can also sporadically check the program/malware to see if it has been modified either automatically by itself or manually by any user(s).

For calculating the hash values (MD5, SHA-1 and SHA256), a self-written bash script will be used, and the checksums will be saved into text file to be compared with the database of VirusTotal.

Below in Table I and Figure 1 are an example for the Malware (504) with its checksums:

\begin{tabular}{|l|l|}
\hline MD5 & a5ce0c535a4f019a60b5be4a662794dd \\
\hline SHA-1 & c593257235490bcd5714016dabce608d3b93429a \\
\hline SHA-256 & 39dcc1164c2a330c5dc32fef185287f496cf13219e48651e59010f9127eb6ef0 \\
\hline
\end{tabular}
TABLE

FILE INFORMATION OF MALWARE (504)

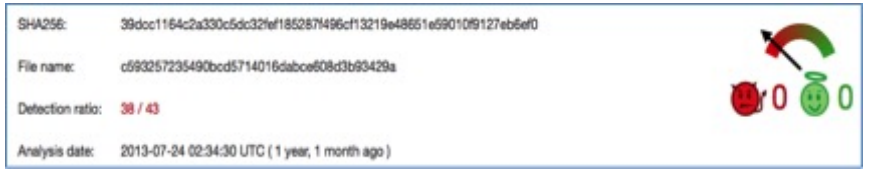

Fig. 1. Detection of Malware (504) via VirusTotal

The overall success rate of all tested cryptographic hashes was $99.88 \%$, as all provided Malware were identified apart from one. Although, the Anti-Virus scanning software identified the Malware(1).sh as thread/malicious program, when comparing the hash values with the VirusTotal database the result was negative (See Table II and Figure 2).

\begin{tabular}{|l|l|}
\hline MD5 & 53188c1c7adba6058a855e24cec42854 \\
\hline SHA-1 & 1112808a0c3c783e423d4d9d3e623783eb2a2712 \\
\hline SHA-256 & 631603fced37bc4da05abbd93d6e1cb76c5e08a1cef129ec3850ae1e495e0e09 \\
\hline
\end{tabular}
TABLE II

FILE INFORMATION OF MALWARE (1).SH

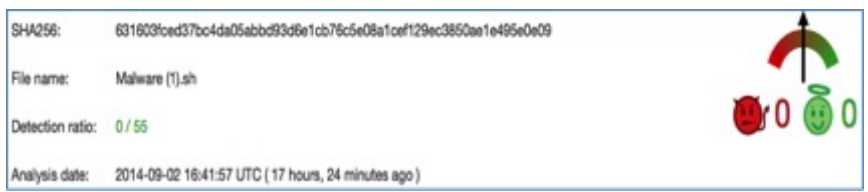

Fig. 2. Detection of Malware (1).sh via VirusTotal

\section{FUZZY HASHING IMPLEMENTATION}

Implementing fuzzy hashing for malware analysis is a similar process to the one used in cryptographic hashes. This means that the fuzzy checksums must be calculated for all 856 understudy malware, and afterwards the checksum of five randomly chosen malwares will be taken and compared with the rest 851 malwares. Doing so, we can detect other malware and identify their similarities.

\section{A. ssdeep}

As mentioned in the literature review the Context Triggered Piecewise Hash (CTPH) or ssdeep calculates a signature (spamsum) for each input file, which can be later used to match these signatures against other file signatures, and to find any possible similarities or matches.

In order to proceed with the comparison we must create the hashes of the 856 Malware using the following command: ssdeepbrTSPY $Y_{Z} B O T$.

The command: ssdeepbrTSPY $Y_{Z} B O T>$ ssdeepHashes.txt enables us to save the hashes on a text file (ssdeepHashes.txt) to be compared with the understudy malware sample.

The hashes of five malwares were randomly chosen and saved into a text to be compared with their signatures in the ssdeepHashes.txt. The malware chosen were: Malware (132), Malware (298), Malware (535), Malware (790), and Malware (803).

After performing the comparison of the signatures between the malwares of the two files, using the command "ssdeep x ssdeepHashes.txt Malware.txt", the following results were received (See Table III):

\begin{tabular}{|l|l|l|}
\hline Malware.txt & ssdeepHashes.txt & Similarity \\
\hline Malware Name & $\boldsymbol{x}$ matches out of 856 & Max 100 \\
\hline Malware (132) & 297 (same matches with Malware 535 and 803) & $97-100$ \\
\hline Malware (298) & 1 & 100 \\
\hline Malware (535) & 297 (same matches with Malware 132 and 803) & $81-100$ \\
\hline Malware (790) & 1 & 100 \\
\hline Malware (803) & 297 Matches (same matches with Malware 132 and 535) & $96-100$ \\
\hline
\end{tabular}

RESULTS OF COMPARING THE FUZZY SIGNATURES USING SSDEEP 
The malware signatures of Malware (298) and (790) were matched only with themselves. The results for the other threemalware signatures that were used to match similarities from the ssdeepHashes.txt ranged a similarity of $96-100 \%$, with a median of $98 \%$. This also means that ssdeep is suitable for matching similarities not only from malware that belong to the same family, but also from emerging families, considering that the matched signatures were the same for all 3 malwares. The only change identified is related to the similarity score. It is worth mentioned here that all matched malware have different Cryptographic Hash value. Below is an example of the first 5 identified similarity matches (See Table IV).

\begin{tabular}{|l|c|c|c|}
\hline Malware & Match & Malware & Similarity \\
\hline Malware.txt:Malware (132) & matches & ssdeepHashes.txt:Malware (504) & 97 \\
Malware.txt:Malware (132) & matches & ssdeepHashes.txt:Malware (249) & 97 \\
Malware.txt:Malware (132) & matches & ssdeepHashes.txt:Malware (629) & 97 \\
Malware.txt:Malware (132) & matches & ssdeepHashes.txt:Malware (340) & 97 \\
Malware.txt:Malware (132) & matches & ssdeepHashes.txt:Malware (844) & 97 \\
$\ldots$ & $\ldots$ & $\ldots$ & $\ldots$ \\
Malware.txt:Malware (803) & matches & ssdeepHashes.txt:Malware (504) & 97 \\
Malware.txt:Malware (803) & matches & ssdeepHashes.txt:Malware (249) & 97 \\
Malware.txt:Malware (803) & matches & ssdeepHashes.txt:Malware (629) & 97 \\
Malware.txt:Malware (803) & matches & ssdeepHashes.txt:Malware (340) & 97 \\
Malware.txt:Malware (803) & matches & ssdeepHashes.txt:Malware (844) & 97 \\
‥ & $\ldots$ & $\ldots$ \\
\hline
\end{tabular}

MALWARE SIMILARITIES WITHIN THREE MALWARE

In order to confirm the above statement, we will use the option "-brp" to recalculate and compare all the spamsums of the malwares provided for this research that belong to same malware family.

Of the 856 Malware identified to belong to the same malware family, only 482 were identified to have some degree of similarity. 364 Malware did not demonstrate any similarity. The similarity among the 482 malware ranged between $30100 \%$, with a median of $67 \%$. Table V shows a sample of the identified similarities:

\begin{tabular}{|l|c|l|c|}
\hline Malware & Match & Malware & Similarity \\
\hline Malware (597) & matches & Malware (180) & 30 \\
Malware (546) & matches & Malware (239) & 30 \\
Malware (180) & matches & Malware (597) & 30 \\
Malware (97) & matches & Malware (55) & 30 \\
Malware (239) & matches & Malware (546) & 30 \\
$\ldots$ & $\ldots$ & $\ldots$ & $\ldots$ \\
Malware (361) & matches & Malware (855) & 100 \\
Malware (332) & matches & Malware (639) & 100 \\
Malware (399) & matches & Malware (757) & 100 \\
Malware (813) & matches & Malware (580) & 100 \\
Malware (725) & matches & Malware (623) & 100 \\
\hline
\end{tabular}

TABLE V

SAMPLE OF SIMILARITIES BASED ON SSDEEP

The results presented by Table $\mathrm{V}$ prove that ssdeep can match similarities from malware that belong to the same malware family, but not necessarily from emerging ones. Furthermore, based on the sample tested, only the malwares with a similarity score of $30 \%$ and above were matched. Finally, the 364 malwares that did not score any similarity, were all files smaller than $100 k b$, which actually confirms that ssdeep is only accurate in identifying similarities in files that are bigger than $100 k b$.

\section{B. sdhash}

The main difference between sdhash and ssdeep, is that sdhash uses Bloom Filters and compares the files using Hamming distance. In order to compare two files with sdhash, , a list of Bloom Filters (.sdbf) of given Malware folder needs to be created. To this end the following command is executed: sdhash -r iMalware folder pathname $i$ i sdhashMalwares.sdbf.

The same command is used for the five Malwares that were used to find similarities by ssdeep. To compare both files 5Malware.sdbf and sdhashMalwares.sdbf, the "-c" option is used.

After comparing the results of the two files, sdhash has matched more similarities compared to ssdeep. The similarity score has ranged from $1100 \%$ (See Table VI). According to Roussev [8], even if the score is 100 , it does not necessarily mean that the two files are exactly identical. However, this is not an issue, as we use sdhash to identify/match similarities and not to identify the exact same files.

\begin{tabular}{|l|c|c|}
\hline 5Malware.sdbf & sdhashMalwares.sdbf & Similarity \\
\hline Malware Name & $\boldsymbol{x}$ matches out of 856 & Max 100 \\
\hline Malware (132) & 755 & $1-100$ \\
\hline Malware (298) & 515 & $1-100$ \\
\hline Malware (535) & 765 & $1-100$ \\
\hline Malware (790) & 506 & $1-100$ \\
\hline Malware (803) & 757 & $1-100$ \\
\hline
\end{tabular}

SDHASH RESULTS OF COMPARING THE 5 MALWARES WITH THE SAMPLE

Although the majority of the matched similarities were ranged from 1 to $56 \%$, sdhash has identified similarities. Furthermore, Roussev [8] claims that the results will be more accurate if we use a threshold of 21 . Therefore, we run the same test with a threshold of 21 . The obtained results are summarized in Table VII.

\begin{tabular}{|l|c|c|}
\hline 5Malware.sdbf & sdhashMalwares.sdbf & Similarity \\
\hline Malware Name & $\boldsymbol{x}$ matches out of $\mathbf{8 5 6}$ & Max 100 \\
\hline Malware (132) & 305 & $34-100$ \\
\hline Malware (298) & 10 & $36-100$ \\
\hline Malware (535) & 305 & $34-100$ \\
\hline Malware (790) & 7 & $35-100$ \\
\hline Malware (803) & 305 & $34-100$ \\
\hline
\end{tabular}

SDHASH RESULTS OF COMPARING THE 5 MALWARES WITH THRESHOLD 21

The identified similarities were less with the threshold 21, but the identified match score was ranged between 99 $100 \%$, which was much higher than before, especially with the Malware (535) and (803). The same test will be conducted now for all Malware. Tables VIII and IX show a sample of the identified similarities without threshold and with a threshold of 21 .

\begin{tabular}{|l|l|c|}
\hline Malware & Malware & Score \\
\hline Malware (0).sh & Malware (10) & 1 \\
Malware (10) & Malware (100) & 1 \\
Malware (100) & Malware (106) & 35 \\
Malware (101) & Malware (107) & 9 \\
Malware (11) & Malware (147) & 18 \\
$\ldots$ & $\ldots$ & $\ldots$ \\
\hline
\end{tabular}

SDHASH RESULTS FROM ALL MALWARE WITHOUT THRESHOLD

sdhash has identified from the 856 Malware 853 (without threshold) with a similarity score ranging between $1100 \%$, and with threshold (21) 795 with the similarity ranging $21100 \%$. The overall results for sdhash were better compared to ssdeep, as sdhash has identified more malware based on their similarity. Apart from that, sdhash has matched similarities to the Malware (0).sh (180\%), that was not identified neither 


\begin{tabular}{|l|l|c|}
\hline Malware & Malware & Score \\
\hline Malware (108) & Malware (158) & 100 \\
Malware (109) & Malware (247) & 53 \\
Malware (11) & Malware (353) & 72 \\
Malware (110) & Malware (450) & 100 \\
Malware (111) & Malware (462) & 42 \\
$\ldots$ & $\ldots$ & $\ldots$ \\
\hline
\end{tabular}

SDHASH RESULTS FROM ALL MALWARE WITH THRESHOLD 21

with the cryptographic hashes or the ssdeep. Table $\mathrm{X}$ shows a sample of similarity matching between Malware (0).sh and other malwares.

\begin{tabular}{|l|l|c|}
\hline Malware & Malware & Score \\
\hline Malware (0).sh & Malware (106) & 35 \\
Malware (0).sh & Malware (189) & 58 \\
Malware (0).sh & Malware (247) & 52 \\
Malware (0).sh & Malware (260) & 59 \\
Malware (0).sh & Malware (353) & 73 \\
$\ldots$ & $\ldots$ & $\ldots$ \\
\hline
\end{tabular}

SDHASH RESULTS FROM ALL MALWARE WITH THRESHOLD 21

\section{C. mvHash}

The mvHash is also a similarity preserving hash (SPH), which is based on the compression technique run length, and it is designed to be an approximate matching algorithm.

Following the same comparison scenario as in ssdeep and sdhash, the results summarized in Table XI were obtained.

\begin{tabular}{|l|c|c|}
\hline Chosen malware & All malwares & Similarity \\
\hline Malware Name & $\boldsymbol{x}$ matches out of $\mathbf{8 5 6}$ & Max 100 \\
\hline Malware (132) & 315 & $3-100$ \\
\hline Malware (298) & 0 & 0 \\
\hline Malware (535) & 182 & $8-100$ \\
\hline Malware (790) & 0 & 0 \\
\hline Malware (803) & 33 & $3-100$ \\
\hline
\end{tabular}

MVHASH RESULTS OF COMPARING THE 5 MALWARES WITH THE SAMPLE

Overall, mvHash has identified and matched 854 out of 856 Malware, with a similarity range score $3100 \%$.

\section{D. $m r s h$ v2}

The last SPH that will be tested is the mrsh. The mrsh (multi-resolution similarity hashing) is powerful variation of the ssdeep. The main difference between these two SPHs is that ssdeep is using the rolling hash, and mrsh is using a polynomial hash - djb2.

Following the same comparison scenario as in ssdeep, sdhash and mvHash, the results summarized in Table XII were obtained.

\begin{tabular}{|l|c|c|}
\hline Chosen malware & All malwares & Similarity \\
\hline Malware Name & x matches out of 856 & Max 100 \\
\hline Malware (132) & 316 & $3-100$ \\
\hline Malware (298) & 1 & 100 \\
\hline Malware (535) & 316 & $3-100$ \\
\hline Malware (790) & 1 & 100 \\
\hline Malware (803) & 316 & $3-100$ \\
\hline
\end{tabular}

TABLE XII

MRSH - V2 RESULTS OF COMPARING THE 5 MALWARES WITH THE SAMPLE

\section{Summary OF Results}

This section will discuss the above results in relation to the identification and similarity rates of each algorithm, as well as the computation time that was required. The results for the fuzzy hashing algorithms are based on using the option against all comparison.

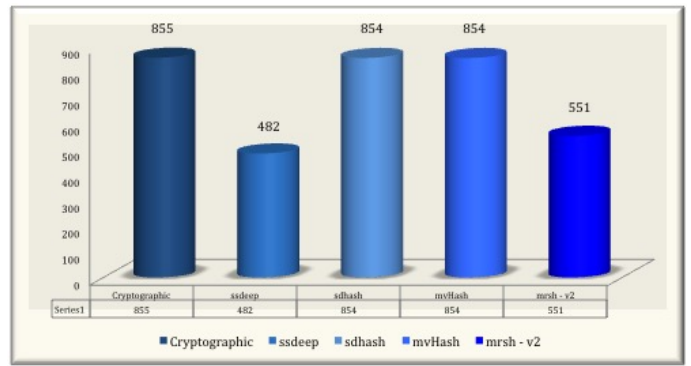

Fig. 3. Identified Malware ( $\times$ out of 856 )

Although, we cannot really compare the functionality of the cryptographic with the fuzzy hashes, it is worth mentioning that the cryptographic hashes have the highest identification rate based on the database of VirusTotal. For fuzzy hashing (see Figure 3), the sdhash and the mvHash have the highest identification rate based on similarities, followed by the mrsh v2 and lastly the ssdeep. A possible explanation for the low ratings of the ssdeep is that the algorithm did not match any malwares that were smaller than $100 k b$, but its computation time was better compared to sdhash and mrsh v2. Furthermore, another explanation for the low results of ssdeep and mrsh v2 is that both of them match similarities only if the digest difference is not too big, and only if they are in the same range.

\begin{tabular}{|c|c|c|c|c|c|c|c|}
\hline \multicolumn{2}{|c|}{ ssdeep } & \multicolumn{2}{c|}{ sdhash } & \multicolumn{2}{c|}{ mvHash } & \multicolumn{2}{c|}{ mrsh - v2 } \\
\hline real & $0 m 18.734 s$ & real & $1 m 22.054 s$ & real & $0 m 4.433 s$ & real & $1 m 17.304 s$ \\
\hline user & $0 m 7.452 s$ & user & $0 m 12.050 s$ & user & $0 m 1.773 s$ & user & $0 m 50.985$ \\
\hline sys & $0 m 6.312 s$ & sys & $0 m 0.751 s$ & sys & $0 m 0.627 s$ & sys & $0 m 0.844 s$ \\
\hline \multicolumn{6}{c}{ TABLE XIII } \\
COMPUTATION TIME
\end{tabular}

According to results summarized in Table XIII, mvHash has the fastest computation time from all fuzzing hashes that were tested, but its identification rate was really low, as it matched $84 \%$ of the TSPY ZBOT malwares with zero similarities, and almost $0 \%(0.01 \%)$ with a similarity match ranging between 4170. The only other fuzzy hashing algorithm that matched the less similarities between all given malware was sdhash, but without using the suggested threshold (i.e., 21). The ssdeep's precursor, the mrsh $\mathrm{v} 2$ had the second largest running time after sdhash, but it matched similarities between the given malware, even to malware that their size was smaller than $100 \mathrm{~kb}$ and were not identified by ssdeep.

Overall, sdhash has outperformed all other fuzzy hashes, but only when used with threshold. The only disadvantage found on sdhash was its running time, but this slight disadvantage can be over-weighted by the main two strengths that relate to the given sdhash's options and its similarity matched accuracy. In other words, sdhash's configuration options make its use much simpler, and the way the results are presented makes the analysis and comparison of the matched malware quite visible the different malware groups, based on their similarity score. This specific option is missing from all other compared hashes, apart from ssdeep. 


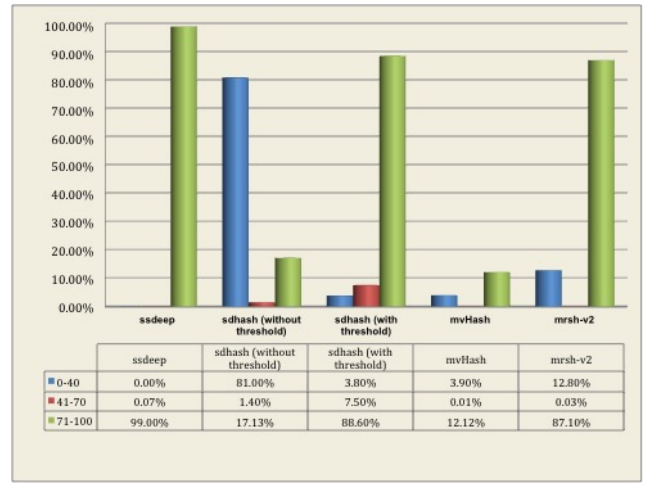

Fig. 4. Similarity Score from each algorithm using Against-All Comparison option

\begin{tabular}{|c|c|c|c|c|}
\hline & ssdeep & sdhash & mvHash & mrsh - v2 \\
\hline Similarity & Frequency & Frequency & Frequency & Frequency \\
\hline 0 & 0 & 0 & 307098 & 0 \\
\hline 5 & 0 & 0 & 11445 & 6404 \\
\hline 10 & 0 & 0 & 2630 & 44 \\
\hline 15 & 0 & 0 & 348 & 35 \\
\hline 20 & 0 & 0 & 4 & 26 \\
\hline 25 & 0 & 149 & 0 & 15 \\
\hline 30 & 3 & 36 & 0 & 8 \\
\hline 35 & 5 & 1579 & 0 & 16 \\
\hline 40 & 4 & 255 & 0 & 1 \\
\hline 45 & 5 & 668 & 0 & 0 \\
\hline 50 & 5 & 9 & 1 & 0 \\
\hline 55 & 3 & 909 & 1 & 2 \\
\hline 60 & 1 & 1237 & 1 & 3 \\
\hline 65 & 7 & 673 & 28 & 2 \\
\hline 70 & 6 & 410 & 2 & 9 \\
\hline 75 & 7 & 964 & 1 & 17 \\
\hline 80 & 5 & 664 & 5 & 17 \\
\hline 85 & 11 & 125 & 3 & 3 \\
\hline 90 & 30 & 67 & 242 & 110 \\
\hline 95 & 440 & 427 & 726 & 654 \\
\hline 100 & 43820 & 43822 & 43504 & 43549 \\
\hline \multicolumn{5}{|c|}{ TABLE XIV } \\
\hline
\end{tabular}

ALl FuZZY HASHES - FREQUENCY OF SIMILARITIES

\section{CONCLUSION}

The implementation of fuzzy hashing can be considered as a successful one in the identification and matching similarities between the same and/or emerging malware families. However, its success depends solely on two factors: first of all, the fuzzy (SPH) algorithm that we are using to identify and match similarities. Secondly and more importantly the hash sample from one or more malware(s), which is required to enable us to use it and find any other similarities. Although, the use of more than one fuzzy hash for malware analysis is recommended to cross-reference the final results, based on this research results the recommended one is sdhash as its results were more accurate compared to all the others that were tested.

Finally, as mentioned above we cannot compare the cryptographic with the fuzzy hashes, but the use of both of them is required for the purpose of malware detection and identification. The use of Cryptographic hashes assists in identifying one known malware. On the other hand, fuzzy hashes can find new malware given the matched similarities.

Furthermore, the effectiveness and feasibility of fuzzy hashing in analysing similarities and malware detection has been this researchs primary objective. This objective was met through the the experiment discussed above. According to the results of all tested fuzzy algorithms, all of them have their strengths and limitations. For example mvHash has the fastest computation time, and its identification rate was similar with the sdhashs. The mrsh-v2 had also a high computation rate, as did the sdhash, and both of them matched a similarity score of $71100 \%$ in $88 \%$ of the identified given malware. The only difference was that sdhash identified similarities in 885 of the 856 malwares, but mrsh v2 presented similarities for only 551 malwares. The only one that identified almost the half of the given malwares was ssdeep, but it has a fast computation time, and the similarities that identified matched a score $71100 \%$.

As a whole fuzzy hashing is the best option for static malware analysis, that does not require a lot of resources, time, and specialist personal. Also with fuzzy hashing it could be possible to identify new malwares, which can be from the same or an emerging malware family, based only on saved fuzzy hashing checksums.

However, fuzzy hashing is weak in identifying similarities in binary packets, and this is a point that requires further research and improvement. Another issue with fuzzy hashing is that at the moment there is no way that we could automatically test if a result is positive or negative, which means that the only safe method is manually. This specific issue could be partially solved, as mentioned above, on condition that a master dataset exists as the one in place for the cryptographic hashes. [17]

\section{REFERENCES}

[1] R. Paleari, "Dealing with next-generation malware," 2011.

[2] M. Lindorfer, C. Kolbitsch, and P. Comparetti, "Detecting environmentsensitive malware," pp. 338 - 357, 2011.

[3] K. Dunham, "A fuzzy future in malware research," 2013.

[4] D. L. Lewis, "The hash algorithm dilemma-hash value collisions," [Online] Available from: http://www.forensicmag.com/print/235. [Accessed: 02 August 2014].

[5] F. Breitinger and H. Baier, "Properties of a similarity preserving hash function and their realization in sdhash," pp. $1-8,2012$.

[6] B. Nelson, A. Phillips, and S. C, "Guide to computer forensic and investigations," Boston: Cengage Learning, 2009.

[7] H. Baier and F. Breitinger, "Security aspects of piecewise hashing in computer forensics," In Proc. of The Sixth International Conference on IT Security Incident Management and IT Forensics (IMF), pp. 21 - 36, May 2011.

[8] V. Roussev, "An evaluation of forensic similarity hashes," digital investigation, vol. 8, pp. $34-41,2011$.

[9] J. Kornblum, "Identifying almost identical files using context triggered piecewise hashing," Digital investigation, vol. 3, pp. 91 - 97, 2006.

[10] C. Negruseri, "Rolling hash, rabin karp, palindromes, rsync and others," [Online] infoarena.ro. Available at: http://www.infoarena.ro/blog/rolling-hash [Accessed: 9 August 2014].

[11] K. Candan and M. Sapino, "Data management for multimedia retrieva," 1st ed. Cambridge University Press, 2010.

[12] J. Oliver, C. Cheng, and Y. Chen, "Tlsh - a locality sensitive hash," pp. $7-13,2013$.

[13] K. Timm, "Malware validation techniques," [online] blogs@Cisco - Cisco Blogs. Available at: http://blogs.cisco.com/security/malware_validation_techniques/ [Accessed 10 Aug. 2014].

[14] F. Breitinger, K. Astebol, H. Baier, and C. Busch, "mvhash-b - a new approach for similarity preserving hashing," In Proc. of The Seventh International Conference on IT Security Incident Management and IT Forensics, 2013.

[15] M. Sikorski and A. Honig, "Practical malware analysis," 1st ed. San Francisco: No Starch Press, 2012.

[16] C. Malin, J. Aquilina, E. Casey, and C. Rose, "Malware forensic field guide for linux systems," 1st ed.

[17] M. Labs, "Threats report fourth quarter 2013," [Online] Available at: http://www.mcafee.com/us/resources/reports/rp-quarterly-threat-q42013.pdf. [Accessed: 05 August 2014]. 